\title{
Salience of physical appearance characteristics among young women in Thailand
}

\author{
Daravan Rongmuang ${ }^{1 *}$ \\ Colleen Corte \\ Linda L. McCreary ${ }^{1}$ \\ Chang G. Park ${ }^{1}$ \\ Arlene Miller ${ }^{2}$ \\ Agatha Gallo ${ }^{3}$
}

\footnotetext{
${ }^{1}$ Department of Health System Science, University of Illinois at Chicago, College of Nursing, United States

2 Department of Community Systems and Mental Health Nursing, Rush University, College of Nursing, United States

${ }^{3}$ Department of Women, Children and Family Health Science, University of Illinois at Chicago, United States ${ }^{*}$ Corresponding author at: Department of Adult and Elderly Nursing, Boromarajonani College of Nursing Suratthani, 84000, Thailand. Tel.: +66 839704697.E-mail addresses: drongm3@uic.edu, daravanr@yahoo.com (D. Rongmuang).
} 


\begin{abstract}
Our aim in the present study was to identify key components of physical appearance among young Thai women. Free listings, focus groups and pile sorting were used. One-hundred twenty young women generated 78 unique physical appearance characteristics. Ninety-four nursing students validated these characteristics in focus groups and then sorted them into piles that reflected separate domains of physical appearance and labeled them. Salience analysis revealed that facial appearance (e.g., bright facial skin, high nose bridge, big eyes) was the most important domain, followed by body weight and shape, skin color and texture, hair (color, texture, length), and 'other' physical appearance (e.g., slender neck, slim fingers). This is the first study to identify aspects of physical appearance that are most salient to young Thai women and that may differ from women in other cultural contexts. These findings could be used to develop culturally grounded measures of physical appearance in Thai women.
\end{abstract}

Keywords: self-concept; identity; body image; cultural issues; qualitative 
Salience of Physical Appearance Characteristics among Young Women in Thailand Physical appearance concerns are prevalent among women in most countries (Cash \& Henry, 1995; Demarest \& Allen, 2000; Hayashi, Takimoto, Yoshita, \& Yoshiike, 2006; Jaeger et al., 2002; Polivy \& Herman, 2004; Wardle, Haase, \& Steptoe, 2006) including Thailand (Thianthai, 2006, 2008). These concerns often lead to negative affect (i.e., depression, low selfesteem) and risky health behaviors (i.e., laxative, diet pill and diuretic use, self-induced vomiting, fasting, and medically unsafe forms of skin-whitening) (Al-Saleh \& Al-Doush, 1997; Counter, 2003; Fuller, 2006; Jung \& Lee, 2006; Jung \& Lennon, 2003; Tiggemann \& Mcgill, 2004). In Western cultures, physical appearance concerns in young women are tied predominantly to body weight and shape (Annis, Cash, \& Hrabosky, 2004; Brown \& Dittmar, 2005; Markus, Hamill, \& Sentis, 1987) with some young women developing a core identity related to body weight and shape that contributes to negative affect (Corte \& Stein, 2005) and disordered eating behaviors (Stein \& Corte, 2007, 2008).

Thai women also appear to be concerned with body weight and shape (Jennings, Forbes, McDermott, Hulse, \& Juniper, 2006; Page \& Suwanteerangkul, 2007; Sritipsukho \& Pacharapaisan, 2004). However, physical appearance concerns among young Thai women extend beyond body weight and shape. Indeed many of these young women engage in a variety of health compromising behaviors aimed at altering different aspects of their physical appearance, including disordered eating behaviors to control body weight and shape (Jennings, Forbes, McDermott, Hulse, \& Juniper, 2006; Page \& Suwanteerangkul, 2007; Sritipsukho \& Pacharapaisan, 2004), use of illegal and unsafe skin whitening products and glutathione injections to lighten the skin (Fuller, 2006; Rush, 2007), and cosmetic surgeries such as double eyelid surgery (blepharoplasty) and nasal bridge augmentation (Aizura, 2009; Saniotis, 2007). .

The diversity of physical appearance-related concerns among Thai women may be in part related to region-specific phenotypic differences. Thailand is a Southeast Asian country that is divided into four main geographic regions: North, Central, North East, and South. People in the 
North generally have lighter skin color than people in the South. In addition to skin tone, facial features also differ by region. People in the North tend to have almond eye shape because most of their ancestors emigrated from China, while people in the North East tend to have wider faces, higher cheekbones, and flatter nose bridges compared to those in other regions (Hesse-Swain, 2006). Women in the Central region (Bangkok) internalize a thin ideal, want to be thin and may take steps to attain such an ideal (Jennings et al., 2006; Rush, 2007; Thianthai, 2006), perhaps because of high media exposure in a metropolitan city where women are more exposed to billboards, wall paintings on the sky train, and cutout advertising presenting the ideal of beauty as thin (Mill, 1997).

Sociocultural factors such as family, pressure from friends, media, cultural norms and economic status may contribute to physical appearance-related concerns among young Thai women (Aizura, 2009; Hesse-Swain, 2006; Swami \& Tovée, 2007; Thianthai, 2006). For example, the numbers of friends who engage in dieting for weight loss and peer pressure to lose weight are factors influencing physical appearance concerns in Thai adolescent young women (Page \& Suwanteerangkul, 2007). Studies have also shown that messages from television and magazines (e.g., advertisements and thin models) that show beautiful tall women with slim bodies, white skin, and preferred facial features are associated with physical appearance dissatisfaction in Thai women (Rush, 2007; Thianthai, 2006).

Cultural beauty norms in Thailand not only place value on thin bodies but extend to other aspects of physical appearance such as light skin (an indicator of higher social class and better education), specific facial features (e.g., an oval face, a high and narrow nose, wide eyes), and being tall (Hesse-Swain, 2006). In Thai culture dark skin is representative of poverty and working class status, while white skin is viewed as representative of wealth and upper class status (Aizura, 2009; Buranasak, 2006; Mills, 1997). Therefore, white skin is viewed as more desirable than dark skin (Rush, 2007). Swami and Tovée (2007) found that socioeconomic status may also influence physical appearance concerns among women, because Thai men of high 
socioeconomic status preferred women with a much lower body mass index compared to men of low socioeconomic status.

Despite the diversity of physical appearance concerns among young Thai women, existing measures of physical appearance tend to focus primarily on body weight and shape. To fill the gap in knowledge about other characteristics that contribute to the overall construct of physical appearance among young Thai women, we conducted an exploratory qualitative research study to identify culturally-relevant terms used to characterize physical appearance among young women in all regions of Thailand. In this article we describe the process used to identify and validate culturally-relevant terms used by young Thai women to describe physical appearance. The terms identified and validated in the present study were subsequently used in a quantitative study using Q-methodology to examine regional differences in the perceived importance of the various domains of physical appearance domains in a different sample of young women in four regions of Thailand (Rongmuang et al., 2011). In that study, we found that women in the North placed the most importance on facial skin (bright face skin); women in the Central region placed the most importance on body weight and shape; women in the Northeast placed the most importance on facial features (oval face, big eyes, and high nose bridge); and women in the South placed the most importance on body skin color (white or white pink [body] skin). Those findings provided evidence that young women from different regions of Thailand place different emphasis on aspects of physical appearance, which supports the need for culturally grounded measures of physical appearance that take into account these regional differences.

The purpose of the present study was to explore and identify the key characteristics of physical appearance among young adult Thai women living in all four regions of Thailand. The following research question was used to guide the present study: "What are the essential and most salient aspects of physical appearance among young women in Thailand?" The findings of the present study together with our study of regional differences in physical appearance among 
young women in Thailand (Rongmuang et al., 2011) will identify the essential characteristics of physical appearance among young Thai women that should be incorporated into valid and reliable measures. The most widely used measures focus primarily on research participants' response to adjectives that describe body weight and shape (Cooper, Taylor, Cooper, \& Fairburn, 1986; Markus, Hamill, \& Sentis, 1987; Stein \& Corte, 2007), but by identifying other salient aspects of physical appearance, this research will serve as the foundation for development of more effective culture-specific measures of physical appearance for Thai women. Measures that tap culture- and region-specific aspects of physical appearance are essential in order to accurately determine factors that cause some women to take on physical appearance as a dominant source of self-definition and engage in health compromising behaviors to alter physical appearance.

\section{Method}

The study was conducted in two phases: Phase 1 involved free listings and Phase 2 involved focus groups and pile sorts with a separate sample of young Thai women. Both Phases 1 and 2 were conducted separately for women in each of the four regions of Thailand (Central, North, North East, and South). A convenience sampling method was used to recruit participants. The inclusion criteria included (a) women aged 18 to 24 years, and (b) born and living in the geographic region of the study site. We purposely limited the age of our sample to women from 18 to 24 for several reasons. First, physical appearance characteristics may be different for young, middle-aged and older women (Demarest \& Allen, 2000). Second, health compromising behaviors to change physical appearance predominantly occur in young women (Jennings et al., 2006; Rudd \& Lennon, 2000; Rush, 2007). Third, the vast majority of other studies focusing on body image, physical appearance concerns, and disordered eating attitudes and behaviors focus on women in specific age ranges (Jennings et al., 2006; Stein \& Corte, 2007, 2008). The study was approved by the Institutional Review Board of the investigators' university, and all participants gave written informed consent. 


\section{Phase 1: Free Listing}

Sample. The participants were 120 young adult women recruited from beauty shops and health clubs. These sites were chosen because they are public places where a wide variety of young women who consider physical appearance to be important may likely be found. We recruited 30 women from each of the four regions of Thailand (Central, North, North East, and South).

Procedure. Participants were asked to list as many Thai words as they could to describe the characteristics of physical appearance in Thai women, alternative terms to describe each characteristic, words to describe their opposites, and alternative terms to describe each opposite word in Thai language. No time limitation was imposed in generating words. Free lists have been recommended as an appropriate method for the first step of research involving the preliminary definition of new cultural domains (Weller \& Romney, 1988). Minimum numbers of 20-30 participants are generally sufficient for reaching saturation when conducting free listings (Weller \& Romney, 1988). In this study free listing was used to generate terms detailing the characteristics of physical appearance among young women in Thailand.

\section{Phase 2: Focus Group Discussion and Pile Sorting}

Sample. The participants were 94 young Thai women, recruited from colleges of nursing under the Ministry of Public Health from all four regions of Thailand. The sites were chosen for participation in focus group discussions and pile sorting because college students, by virtue of their educational status, may be better able than women from the general population to conceptualize and articulate different domains of physical appearance and to perform the abstract task of categorizing the specific listed characteristics into these domains. Nursing students (rather than other college students) were sampled specifically because they are predominantly women.

Focus group discussion procedure. The focus group discussions were led by the first author. Twelve focus group discussions were conducted, with the groups ranging in size from 7- 
9 participants per group. Three focus groups were conducted in each of the four regions of Thailand. The focus group discussions lasted for approximately one hour. The discussions were conducted in the official national language (hereafter referred to as Standard Thai). The participants were asked to generate words that describe physical appearance characteristics in Thai women, their alternatives, opposite words, and their alternative terms of each opposite word. During the tape recorded focus group discussions, the participants talked freely and spontaneously about the characteristics of physical appearance in Thai women, alternative terms to describe each construct, and words to describe their opposites in Thai women. These terms were written on flip charts, and field notes were made during the focus group discussions. The purpose of the focus group discussions was to validate the words generated by individuals in Phase 1 of the study. In addition to enhancing content validity, this approach strengthened credibility of the findings by triangulating responses from two different levels of respondents (i.e., individual level for free listings and group level for focus group discussions) to identify the attributes of physical appearance among Thai women (Patton, 2002).

The first author fully transcribed all tapes from the focus group discussions. Before starting the analysis, she reviewed all transcripts along with field notes and flip charts to check for accuracy. Content analysis was used to explore the characteristics of physical appearance and the components of physical appearance among young adult Thai women. Particular attention was paid to specific words and phrases used by participants. Relevant verbatim quotations from participants were selected to support the conclusions. All quotations were translated verbatim from Standard Thai to English by the first author, who is bilingual with Thai as the first language. To validate the translations of Thai quotes to English, a linguistic scholar who is bilingual with English as her first language and Thai as her second language read all quotations and verified the accuracy of the translations. The first author and the linguistic scholar discussed the use of appropriate vocabularies and sentence structure. 
Pile sorting procedure. Simple pile sorts were conducted with the participants who participated in the focus group discussions. The process lasted for approximately 30 minutes. All of the 78 words generated in the free listings describing the characteristics of physical appearance in Thai women and validated in focus group discussions were used in the pile sorting. Each group of participants was asked to categorize the characteristics of physical appearance in Thai women into distinct physical appearance domains. They were then asked to rank-order the importance of the domains from most important to least important. Pile sorts are a commonly used method to study categorizations of responses used by the participants and to identify superordinate categories. This method allows the participants to sort items into categories according to their own views and eliminates the biases stemming from researchers categorizing the items from their perspectives (Weller \& Romney, 1988).

The researcher placed the cards (each with a physical descriptor written on it) on the table in random order. Participants worked as a group (of 7 or 9) and sorted the physical appearance characteristics into piles representing categories or domains of physical appearance that made sense to them. They were instructed that they could make as many or as few piles representing categories as needed. If a card could fit in multiple piles, the group was instructed to pick one of the piles that they felt made the most sense. After the cards were sorted into piles, participants were asked to name each of the categories. Finally, participants were asked to prioritize the piles in terms of their order of importance.

\section{Results}

\section{Free Listing}

A total of 120 young adult women took part, ranging in age from $18-24(M=21.13, S D=$ 1.56). Young women across all four regions of Thailand generated a total of 78 unique physical appearance characteristics; women across all four regions of Thailand generated similar content in the free listing procedure. The minimum number of characteristics generated by any one individual was 32 and the maximum number was 77 . The average number was 48 physical 
appearance characteristics. There were 46 characteristics that were listed by more than $50 \%$ of the participants; these were considered the core physical appearance characteristics for young Thai women (see Table 1).

We used a method called salience analysis (Smith \& Borgatti, 1998) to identify the most salient characteristics among young women in each region. First, for each participant, we determined the salience of the characteristics by ranking characteristics inversely (final characteristic listed was assigned a value of one, and each previous characteristic was assigned a value of 2,3 , 4, etc.), based on the assumption that the characteristic listed last was least important to the participant. For each individual, the rank assigned to each characteristic was divided by the total number of characteristics they listed. Second, we calculated a mean salience value for each of the characteristics across all 30 women within a region by summing the salience scores for each characteristic across each participant and dividing by 30 (the total number of women in each region).

Table 2 shows the top 10 physical appearance characteristics ranked by salience. Across all four regions, the range of mean salience of physical appearance characteristics were as follows: shapely (range: .95-.97), bright [radiant] facial skin (range: .93-.97), high nose bridge (range: .76-.96), white skin (range: .82-.95), oval face (range: .88-.95), and big eyes (range: .80.90). For young women in the Central and the South regions of Thailand, shapely was the most salient characteristic, whereas among women in the North and the North East regions, it was the second most salient. For women in the North, bright facial skin was the most salient characteristic. High nose bridge was the most salient characteristic for women in the North East. The mean salience of white skin was highest among women in the South compared to those in other regions.

\section{Focus Group Discussion}

A total of 94 young adult women in all four regions women took part, ranging in age from 18-22 $(M=20.29, S D=1.02)$. In each region, young women in the focus group discussions 
added no further physical appearance characteristics to those already generated by young women in the free listings, but they eliminated some terms that are not used in Standard Thai or are region-specific terms. For example, the Standard Thai term, jam mam ("chubby") is used to describe children but would not be used in Standard Thai to describe a young woman's physical appearance. Region-specific terms, such as the Northeastern Thai phrase dang mab ("flat nose bridge") were deleted, as were slang terms such as e dam ("black girl”), or dam tap pet (“black like a duck's liver").

Focus group discussions also provided more detailed information about the characteristics. For example, the participants provided detailed descriptive definitions for "bright-skinned face"; this term meant not only having luminous facial skin, but also included having a combination of white-pink colored facial skin, skin without dark spots or pimples, and baby-face skin. In another example, participants described the meaning of big eyes. As one participant said "Many people (women) want double-eyelid surgery to make their eyes bigger and wider. Women who have big and round-shaped eyes look beautiful." In describing a high nose bridge, one participant said,

I saw my friend who had nose augmentation. She looks more beautiful now. However, some people had surgery, but they don't look good because they made the [nose] bridge too high. I think Thai women want to have a high nose bridge (just not too high) and narrow nasal base. We don't want it too high like Farang ("Westerner"). If it is high, it will not look natural. People may know that we have had surgery. When we take pictures, we can see the nose looks more prominent.

Women identified "shapely" as another important characteristic of physical appearance. In yet another example, one participant said "Shapely is not skinny. Women who are shapely have to have full breasts, narrow waist, and beautiful hips (not too big, but those that look good when they wear jeans)." 


\section{Pile Sorting}

The participants identified five domains of physical appearance including: facial appearance, body weight and shape, skin, hair, and other physical appearance. The same five domains of physical appearance were identified by women in all four regions. After content domains were identified, participants were asked to rank the domains in order of importance. We again used salience analysis (Smith \& Borgatti, 1998) to identify the most salient domains (rather than individual characteristics) of physical appearance among Thai women. First, we determined the salience of the five domains for each focus group by ranking domains inversely (final domain listed equals one, and domains increase by one moving up the list of five domains) and dividing by the total number of domains (5). Second, we calculated a mean salience value for each of the five domains by summing the salience scores for each of the domains across each of the focus groups and dividing by the total number of focus groups (12).

Table 3 shows the perceived importance for each domain for each of the 12 focus groups. Facial appearance was the most important domain for Thai women (mean salience $=.97$ ). The second most important was body weight and shape (mean salience $=.92$ ). The third most important was skin (mean salience $=.68$ ). The fourth most important was hair (mean salience $=$ .47 ), followed by other physical appearance (mean salience $=.22$ ). The five most frequently listed physical appearance characteristics in each domain of importance are shown in Table 4.

\section{Discussion}

In this study, we used free listings, focus group discussions, and pile sorting processes to explore the key characteristics and the perceived importance of the domains of physical appearance among young adult Thai women in the four regions of Thailand (Central, North, North East, and South). The most notable finding was that women in all four regions generated characteristics in the same five distinct domains of physical appearance. This finding is consistent with Cash (2005) who suggested that physical attractiveness is associated with many physical standards that go beyond thinness. Although there were some regional differences in the 
salience of particular physical appearance characteristics, the same characteristics were generated by women in all four regions of Thailand.

Facial appearance was identified as the most important domain, followed by body weight and shape, skin, hair, and other physical appearance. Our findings are consistent with the few other existing studies that show that Asian women not only place importance on body weight, but also place importance on facial features (Jackson \& Chen, 2007). The most frequently listed characteristics related to facial appearance were oval face, bright facial skin, big eyes, high nose bridge, and beautiful lips. Thianthai (2008) similarly found that Thai women place importance on the appearance of their nose, eyes, and overall facial structure. More specifically, they desire big eyes, high nose bridge and narrow nasal base. This may relate to Thai people tending to have round facial shapes, flat nose bridges, wide nasal bases, and for those Thai people whose ancestors migrated from China, almond-shaped eyes.

Another important feature of facial appearance is "bright face skin" which Thai women described as luminous white facial skin. This is consistent with a previous study of Thai women that showed that the meaning of brightness is related to whiteness, freshness, shining, and no darkness (Buranasak, 2006). Valuing of light-colored facial skin is shared by other Asian cultures. Like Thai women, Japanese women also use expensive face whitening products to make their face skin lighter, and they tend to stay away from the sunlight to avoid tanning in order to maintain their beauty (Ashikari, 2005). Similarly, in China, women prefer a light facial skin color (typically a light pink color) (Lee, Leung, Lee, Yu, \& Leung, 1996). It is noteworthy that Thai women identified facial skin color and body skin color as separate domains of physical appearance. This may be because of the enormous media influence about facial skin whitening products (Rush, 2007; Thianthai, 2006).

Our findings suggest that body weight and shape are very important for Thai women. This finding is consistent with the findings of other studies that show that Thai women desire to be thin and most are dissatisfied with their body weight and shape (Jennings et al., 2006; Sharps, 
Price-Sharps, \& Hanson, 2001). The terms Thai women used to characterize body weight and shape in this study were shapely, skinny, flat belly, small waist, and full breasts. Thai women preferred being shapely rather than skinny, because they defined skinny as bony. However, Thai women considered shapely women to have a considerably smaller body size compared to women in the West, which is consistent with the preferences of Chinese women (Lee et al., 1996). Japanese women also desire to be thin and are very concerned with their weight (Kaneko, Kiriike, Ikenaga, Miyawaki, \& Yamagami, 1999).

These results show similarities between Thai women and Caucasian-Americans, Hispanic-Americans, African-Americans and Asian-Americans, who also indicate a desire to be thin or thinner than their actual body size (Altabe, 1998). However, studies of women in the West have found that breast size, waist circumference, and muscle tone are the major concerns related to physical appearance (Miller et al., 2000; Rudd \& Lennon, 2000; Wiederman \& Hurst, 1998). A study by Altabe (1998) showed that Caucasian-American and African-American women listed muscle tone as one of the most commonly desired physical appearance characteristics. In our study, characteristics related to muscle tone such as firm and fit were not spontaneously generated by young Thai women. When asked in the focus groups whether characteristics about muscle tone should be included, the women indicated that these characteristics were not desirable in Thai women.

Skin color was identified as the third most important domain of physical appearance for Thai women. The main distinction between physical appearance components in Thai women and Caucasian women is that Caucasian women want to be tan (Lazovich et al., 2004) and many are willing to undergo harmful ultraviolet light exposure and risk developing skin cancer to achieve tan skin. On the other hand, Thai women desire to have lighter skin (Thianthai, 2008) and to achieve this, many are willing to risk their health or life through the use of harmful products. Older people often describe women moving into Bangkok (the capital city of Thailand) as "going to get [white] skin" (Mill, 1997, p. 43). Normally, Thai women tend to have light brown to dark 
brown skin, but even light brown skin is sometimes described as black skin, which Thai women tend to consider unattractive (Rush, 2007; Thianthai, 2006). In Thailand, commercial advertising media and film media present the image of ideal Thai women as one with white skin-which is not only perceived as beautiful, but also a reflection of high class and modern womanhood in Thai society. A popular Chinese quote cited in Lee et al. (1996, p. 83) states that "a light complexion can conceal extreme ugliness." This is a sentiment that echoes across Thailand as well. These differences in skin color preference between women in Thailand and women in the West clearly illustrate that physical appearance characteristics are culture-specific.

Hair was the fourth most important physical appearance domain listed for Thai women, with long black hair being most desirable. Characteristics of hair, both similar and different, are also salient among women of other races and cultures. In Chinese culture, dense black hair is a defining characteristic of beauty for women (as cited in Lee et al., 1996). Asian-Americans listed brown and long hair as the ideal for women (Altabe, 1998). African-American women prefer long, straight hair because it is easier to manage and style than short, curly hair (Alexander, Reid, \& Sarin, 2007). Hispanic-American listed brown hair as the ideal characteristic, whereas Caucasian- Americans prefer blonde hair (Altabe, 1998).

Other physical characteristics were also important to Thai women. Thai women view their bodies in detail; for example, they frequently referenced finger size and slenderness of fingers, and slenderness of the neck as important indicators of beauty. Our findings are similar to those found by Thianthai (2008) and are consistent with an ancient Chinese saying that women with fine fingers are "like spring onion" -- an indication of feminine beauty (as cited in Lee et al., 1996, p. 78). The variety of words used to describe physical characteristics, together with the identification of five important domains of physical appearance that have varying degrees of importance across the four regions of Thailand, provide empirical evidence of the need to go beyond body weight and shape when assessing the perceptions of young Thai women about their physical appearance. Identification of the specific aspects of physical appearance that are 
deemed important to young Thai women is an important first step toward not only measuring how Thai women identify themselves, but also targeting the primary risk behaviors that should be addressed in health promotion interventions.

Young Thai women's perceptions of discrepancy between their actual physical appearance and the Thai cultural ideal of beauty may lead to low self-esteem, depression, body dissatisfaction, eating disorder behaviors, use of dangerous or unsafe form of skin whitening products, and cosmetic surgery to alter physical appearance (Al-Saleh \& Al-Doush, 1997; Cooley \& Toray, 2001; Corte \& Stein, 2005; Counter, 2003; Fuller, 2006; Jung \& Lee, 2006; Jung \& Lennon, 2003; Rush, 2007; Stein \& Corte, 2007,2008; Tiggemann \& Mcgill, 2004). Future research is needed to test these relationships and develop interventions to prevent their negative outcomes. Factors that contribute to unrealistic standards of beauty among Thai women need to be identified so that effective interventions can be developed. In the US, Stein's work with eating disordered women shows that deficits in identity development contribute to body weight and shape becoming a dominant source of self-definition, and that interventions to build new positive domains of self-definition and a stronger sense of self lead not only to reduction of eating disorder symptoms, but also to increased functional health and well-being (Stein, Wing, Corte, \& Nuliyala, 2011). Similar research using culturally grounded measures and interventions may be beneficial in promoting health among young Thai women.

The limitations of this study are primarily related to sampling issues. First, the convenience sampling method yielded a relatively homogeneous sample of young urban women who are able to afford to frequent beauty salons and health clubs and attend universities. These young women may differ in socioeconomic status and in other ways from young Thai women as a group, thus limiting the ability to generalize from these qualitative findings. As Swami and Tovée (2007) noted, socioeconomic status has a strong influence on physical appearance ideals among Thais. Second, though we recruited women from all 4 regions of Thailand, the small sample size limits our generalizability. Third, because our age range was limited to women 
between 18 - 24 years of age, our findings may not apply to Thai women younger than 18 and/or older than 24. Fourth, we recruited different types of samples (women from beauty shops and fitness centers for Phase 1 and nursing students for Phase 2) for the different phases of the study which could be a strength, but could also be a limitation. While we recruited women at beauty shops and fitness centers because we thought they would be likely to consider physical appearance important, women at beauty shops and fitness centers may not be representative of all or even most Thai young women.

College students were recruited in Phase 2 of the study because we thought that because of their educational status they may be more able to conceptualize domains of physical appearance from the physical appearance characteristics generated by the women from beauty shops and fitness centers in Phase 1 of the study, but we do not have any empirical evidence that this is true. In addition, we used nursing students because they are predominantly women, yet nursing students may not be representative of other college students or young Thai women. A final limitation of the study is related to the fact that many of the terms used by participants are highly abstract concepts that can vary greatly in meaning from person-to-person (e.g., "beautiful" lips, "beautiful" hips). Additional studies should seek to more precisely define these concepts and terms.

This is the first study to identify the unique physical appearance characteristics important to young women in Thailand. Future research is needed to develop a culturally grounded measure of physical appearance in Thai women that can be used in other studies. This study yielded a culturally specific core set of physical appearance characteristics and provides essential foundational data for the development of culturally grounded measures to assess physical appearance among Thai women. Ultimately, identification of these key characteristics can inform more culturally relevant interventions to diminish the perceived importance of physical appearance to young Thai women's overall sense of self. Such prevention and health promotion interventions may empower young women to build a strong identity, reduce the use of extreme 
health-compromising behaviors to enhance physical appearance, and adopt a more healthy life style. 


\section{References}

Aizura, A. Z. (2009). Where health and beauty meet: Femininity and Racialisation in Thai cosmetic surgery clinics. Asian Studies Review, 33, 303-317. doi:10.1080/10357820903153707

Alexander, M., Reid, T., \& Sarin, H. (2007). Optimization of Hair Beauty in African-American Women (Research Report ME 555-07-01). Retrieved from http://eode.engin.umich.edu/POD2/papers/2007/ME555-07-01-FinalReport.pdf

Al-Saleh, I., \& Al-Doush, I. (1997). Mercury content in skin-lightening creams and potential hazards to the health of Saudi women. Journal of Toxicology and Environment Health, 51, 123-130. doi: 10.1080/00984109708984016

Altabe, M. (1998). Ethnicity and body image: quantitative and qualitative analysis. International Journal of Eating Disorders, 23, 135-159. doi:10.1002/(SICI)1098108X(199803)23:2<153::AID-EAT5>3.0.CO;2-J

Annis, N. M., Cash, T. F., \& Hrabosky, J. (2004). Body image and psychosocial differences among stable average weight, currently overweight, and formerly overweight women: the role of stigmatizing experiences. Body Image, 1, 155-167.

doi:10.1016/j.bodyim.2003.12.001

Ashikari, M. (2005). Cultivating Japanese whiteness: The whitening cosmetics boom and the Japanese identity. Journal of Material Culture, 10, 73-91. doi: $10.1177 / 1359183505050095$

Brown, A., \& Dittmar, H. (2005). Think thin and feel bad: the role of appearance schema activation, attention level, and thin-ideal internalization for young women's responses to 
ultra-thin media ideals. Journal of Social and Clinical Psychology, 24, 1088-1113. doi:10.1521/jscp.2005.24.8.1088

Buranasak, J. (2006). The whitening cream influences self and identity of female workers (Master's thesis). Retrieved from http://mulinet10.li.mahidol.ac.th/e-thesis/4437082.pdf

Cash, T. F. (2005). The influence of sociocultural factors on body image: Searching for constructs. Clinical Psychology Science and Practice, 12, 438-442. doi: 10.1093/clipsy.bpi055

Cash, T. F., \& Henry, P. E. (1995). Women's body image: The results of a national survey in the U.S.A. Sex Role, 33, 19-28. doi: 10.1007/BF01547933

Cooley, E., \& Toray, T. (2001). Body image and personality predictors of eating disorder symptoms during the college years. International Journal of Eating Disorders, 30, 28-36. doi: 10.1002/eat.1051

Corte, C., \& Stein, K. F. (2005). Body weight self-schema: Determinant of mood and behavior in women with an eating disorder, Journal of Applied Social Psychology, 35, 1698-1718. doi:10.1111/j.1559-1816.2005.tb02191.x

Counter, S. A. (2003, December 16). Whitening skin can be deadly. The Boston Globe. Retrieved from http://www.boston.com

Cooper, P. J., Taylor, M. J., Cooper, Z. \& Fairburn, C. G. (1986). The development and validation of the Body Shape Questionnaire. International Journal of Eating Disorders, $6,485-494$.

Demarest, J., \& Allen, R., (2000). Body image: Gender, ethnic, and age differences. The Journal of Social Psychology, 140, 465-472. doi:10.1080/00224540009600485 
Fuller, T. (2006, May 14). A vision of pale beauty carry risks for Asia's women. The New York Times. Retrieved from http://www.nytimes.com

Hayashi, F., Takimoto, H., Yoshita, K., \& Yoshiike, N. (2006). Perceived body size and desire for thinness of young Japanese women: A population-based survey. British Journal of Nutrition, 96, 1154-1162. doi: 10.1017/BJN20061921

Hesse-Swain, C. (2006). Programming beauty and the absence of Na Lao: Popular Thai TV and identity formation among youth in Northeast Thailand. GeoJournal, 66, 257-272. doi: $10.1007 / \mathrm{s} 10708-006-9028-\mathrm{x}$

Jackson, T. \& Chen, H. (2007). Identifying the eating disorder symptomatic in China: The role of sociocultural factors and culturally defined appearance concerns. Journal of Psychosomatic Research, 62, 241-249. doi:10.1016/j.jpsychores.2006.09.010

Jaeger, B., Ruggiero, G. M., Edlund, E., Gomez-Perretta, C., Lang, F., Mohammadkhani, P., ...Lamprecht, F. (2007). Body dissatisfaction and its interrelations with other risk factors for bulimia nervosa in 12 countries. Psychotherapy and Psychosomatics, 71, 54-61. doi: $10.1159 / 000049344$

Jennings, P. S., Forbes, D., McDermott, B., Hulse, G., \& Juniper, S. (2006). Eating disorder attitudes and psychopathology in Caucasian Australian, Asian and Australian and Thai university students. Australian and New Zealand Journal of Psychiatry, 40, 143-149. doi:10.1111/j.1440-1614.2006.01761.x

Jung, J., \& Lee, S-H. (2006). Cross-cultural comparisons of appearance self-schema, body image, self-esteem, and dieting behavior between Korean and U.S. women. Family and Consumer Research Journal, 34, 350-365. doi:10.1177/1077727X06286419 
Jung, J., \& Lennon, S. J. (2003). Body image, appearance self-schema, and media images. Family and Consumer Sciences Research Journal, 23, 27-51. doi: $10.1177 / 1077727 X 03255900$

Kaneko, K., Kiriike, N., Ikenaga, K., Miyawaki, D., \& Yamagami, S. (1999). Weight and shape concerns and dieting behaviors among pre-adolescents and adolescents in Japan. Psychiatry and Clinical Neurosciences, 53, 365-371. doi:10.1046/j.14401819.1999.00559.x

Lazovich, D., Forster, J., Sorensen, G., Emmons, K., Stryker, J., Demierre, M-F., Hickle, A., \& Remba, N. (2004). Characteristics associated with use or intention to use indoor tanning among adolescents. Archives of Pediatrics and Adolescent Medicine, 158, 918-924. doi:10.1001/archpedi.158.9.918

Lee, S., Leung, T., Lee, A. M., Yu, H., \& Leung, C. M. (1996). Body dissatisfaction among Chinese undergraduates and its implications for eating disorders in Hong Kong. International Journal of Eating Disorders. 20, 77-84. doi:10.1002/(SICI)1098108X(199607)20:1<77::AID-EAT9>3.0.CO;2-1

Markus, H., Hamill, R., \& Sentis, K. P. (1987). Thinking fat: Self-schemas for body weight and the processing of weight relevant information. Journal of Applied Social Psychology, 17, $50-71$.

Mill, M. B. (1997). Contesting the margins of modernity: Women, Migration, and consumption in Thailand. E-Journal of American Ethnologist, 24, 37-61. Retrieved from http://www.jstor.org/stable/pdfplus/646565.pdf

Miller, K. J., Gleaves, D. H., Hirsch, T. G., Green, B. A., Snow, A. C., \& Corbett, C. C. (2000). Comparison of body image dimensions by race/ethnicity and gender in a university 
population. International Journal of Eating Disorders, 27, 310-316.

doi:10.1002/(SICI)1098-108X(200004)27:3<310::AID-EAT8>3.0.CO;2-Q

Page, R. M. \& Suwanteerangkul, J. (2007). Dieting among Thai adolescents: Having friends who diet and pressure to diet. Eating and Weight Disorder, 12, 114-124.

Patton, M. Q. (2002). Qualitative research and evaluation methods, $3^{\text {rd }}$ ed. Thousand Oaks, CA: Sage.

Polivy, J., \& Herman, C, P. (2004). Sociocultural idealization of thin female body shapes: An introduction to the special issue on body image and eating disorders. Journal of Social and Clinical Psychology, 23, 1-6. doi: 10.1521/jscp.23.1.1.26986

Rongmuang, D., McElmurry, B. J., McCreary, L. L., Park, C. G., Miller, A. G., \& Corte, C. (2011). Regional differences in physical appearance identity among young adult women in Thailand. Western Journal of Nursing Research, 33, 106-120. doi: $10.1177 / 0193945910381762$

Rudd, N. A., \& Lennon, S. J. (2000). Body image and appearance-management behaviors in college women. Clothing and Textiles Research Journal, 18, 152-162. doi:10.1177/0887302X0001800304

Rush, E. (2007). A reflective analysis of a transformative pedagogical approach at a rural Thai university (Master's thesis). Retrieved from http://wwwlib.murdoch.edu.au/adt/browse /view/adt-MU20071129.122953

Saniotis, A. (2007). Changing ethics in medical practice: A Thai perspective. Indian Journal of Medical Ethics, 4, 24-25. 
Sharps, M. J., Price-Sharps, J. L., \& Hanson, J. (2001). Body image preference in the United States and rural Thailand: An exploratory study. The Journal of Psychology, 135, 518526. doi:10.1080/00223980109603716

Smith, J. J. \& Borgatti, S. P. (1998). Salience counts-and so does accuracy: Correcting and updating a measure for free-list-item salience. Journal of Linguistic Anthropology. 7, 208-209. doi:10.1525/ilin.1997.7.2.208

Sritipsukho, P., \& Pacharapaisan, N. (2004). Inappropriate weight management among Thai women consuming anorectics prescribed by private clinics in Bangkok. Journal of the Medical Association of Thailand, 87, 1477-81

Stein, K. F., \& Corte, C. (2007). Identity impairment and the eating disorder: content and organization of the self-concept in women with anorexia nervosa and bulimia nervosa. European Eating Disorders Review, 15, 58-69. doi:10.1002/erv.726

Stein, K. F., \& Corte, C. (2008). The identity impairment model: A longitudinal study of selfschemas as predictors of disordered eating behaviors. Nursing Research, 57, 182-190. doi:10.1097/01.NNR.0000319494.21628.08

Stein, K.F., Wing, J., Corte, C., \& Nuliyala, U. (2011). A randomized clinical trial of an identity intervention program for women with eating disorders. Manuscript submitted for publication.

Swami, V., \& Tovée, M. J. (2007). Differences in attractiveness preferences between observers in low and high-resource environments in Thailand. Journal of Evolutionary Psychology, 5, 149-160. doi:10.1556/JEP.2007.1005

Thianthai, C. (2006). Influential sources affecting Bangkok adolescent body image perceptions. International Journal of Adolescent Medicine and Health, 18, 633-641. 
Thianthai, C. (2008). Do male and female adolescents view their dissatisfaction with body parts in the same way?. International Journal of Adolescent Medicine and Health, 20, 33-39.

Tiggemann, M. \& Mcgill, B. (2004). The role of social comparison in the effect of magazine advertisements on women's mood and body dissatisfaction. Journal of Social and Clinical Psychology, 23, 23-44. doi:10.1521/jscp.23.1.23.26991

Wardle, J., Haase, A. M., \& Stepto, A. (2006). Body image and weight control in young adults: international comparisons in university students from 22 countries. International Journal of Obesity, 30, 644-651. doi:10.1038/sj.ijo.0803050

Weller, S. C., \& Romney, A. K. (1988). Systematic data collection. Newbury Park, CA: Sage.

Wiederman, M. W., \& Hurst, S. R. (1998). Body size, physical attractiveness, and body image among young adult women: Relationships to sexual experience and sexual esteem. The Journal of Sex Research, 35, 272-281. doi:10.1080/00224499809551943 